\title{
Reversible Control of Gelatin Hydrogel Stiffness using DNA Crosslinkers
}

Alex Buchberger, ${ }^{1,2 \ddagger}$ Harpinder Saini, ${ }^{3 \neq}$ Kiarash Rahmani Eliato, ${ }^{4,5,6 \neq}$ Ryan Merkley, ${ }^{1,2}$ Yang Xu, ${ }^{1,2}$ Azadeh Zare, ${ }^{4,5,6}$ Julio Bernal, ${ }^{1,2}$ Robert Ros, ${ }^{4,5,6 *}$ Mehdi Nikkhah, ${ }^{3 *}$ Nicholas Stephanopoulos ${ }^{1,2 *}$

( ${ }^{\ddagger}$ these authors contributed equally)

( robert.ros@asu.edu, mnikkhah@asu.edu,nstepha1@asu.edu)

1. School of Molecular Sciences, Arizona State University, Tempe AZ

2. Center for Molecular Design and Biomimetics, The Biodesign Institute, Arizona State University, Tempe AZ

3. School of Biological and Health Systems Engineering, Arizona State University, Tempe $A Z$

4. Department of Physics, Arizona State University, Tempe, AZ

5. Center for Biological Physics, Arizona State University, Tempe, AZ

6. Center for Single Molecule Biophysics, The Biodesign Institute, Arizona State University, Tempe AZ

\section{Abstract}

Biomaterials with dynamically tunable properties are critical for a range of applications in regenerative medicine and basic biology. In this work, we show the reversible control of gelatin methacrylate (GeIMA) hydrogel stiffness through the use of DNA crosslinkers. We replaced some of the inter-GelMA crosslinks with double-stranded DNA, allowing for their removal via toeholdmediated strand displacement. The crosslinks could be restored by adding fresh dsDNA with complementary handles to the hydrogel. The elastic modulus ( $\left.G^{\prime}\right)$ of the hydrogels could be tuned between 500 and $1000 \mathrm{~Pa}$, reversibly, over two cycles without degradation of performance. By functionalizing the gels with a second DNA strand, it was possible to control the crosslink density and a model ligand in an orthogonal fashion with two different displacement strands. Our results demonstrate the potential for DNA to reversibly control both stiffness and ligand presentation in a protein-based hydrogel, and will be useful for teasing apart the spatiotemporal behavior of encapsulated cells.

The ability to dynamically remodel the extracellular matrix (ECM) during the process of development, tissue regeneration, or wound healing is a hallmark of biological systems. As a result, there has been an explosion of interest in developing biomaterials that can recapitulate this temporal control-in both ligand presentation and mechanical properties (e.g. stiffness)—in order to guide cell behavior. Dynamic changes in the ECM have also been observed in malignancies such as cancer, where an increase in stromal matrix stiffness leads to tumor growth, cancer cell invasion, and reduced drug efficacy. ${ }^{1-7}$ Thus, hydrogels that can mimic these changes are key for dissecting the influence of matrix properties on these crucial biological functions. ${ }^{8}$ Most dynamic materials designed to date involve the irreversible breakage of ligand-matrix bonds or hydrogel crosslinks, for example via photocleavable moieties. ${ }^{9}$ Although these approaches are useful for probing the biological effects of a one-time change in the ECM, they do not allow for reversibility and cannot (with a few exceptions ${ }^{10}$ ) control multiple properties independently.

In recent years, a number of reversible molecular mechanisms have been introduced into biomaterials. ${ }^{11}$ One attractive option is the use of oligonucleotides like DNA and PNA, as either tethers for bioactive signals, or as hydrogel crosslinks. Advantages of using DNA for these purposes include sequence-specificity-which allows for orthogonal control over multiple 


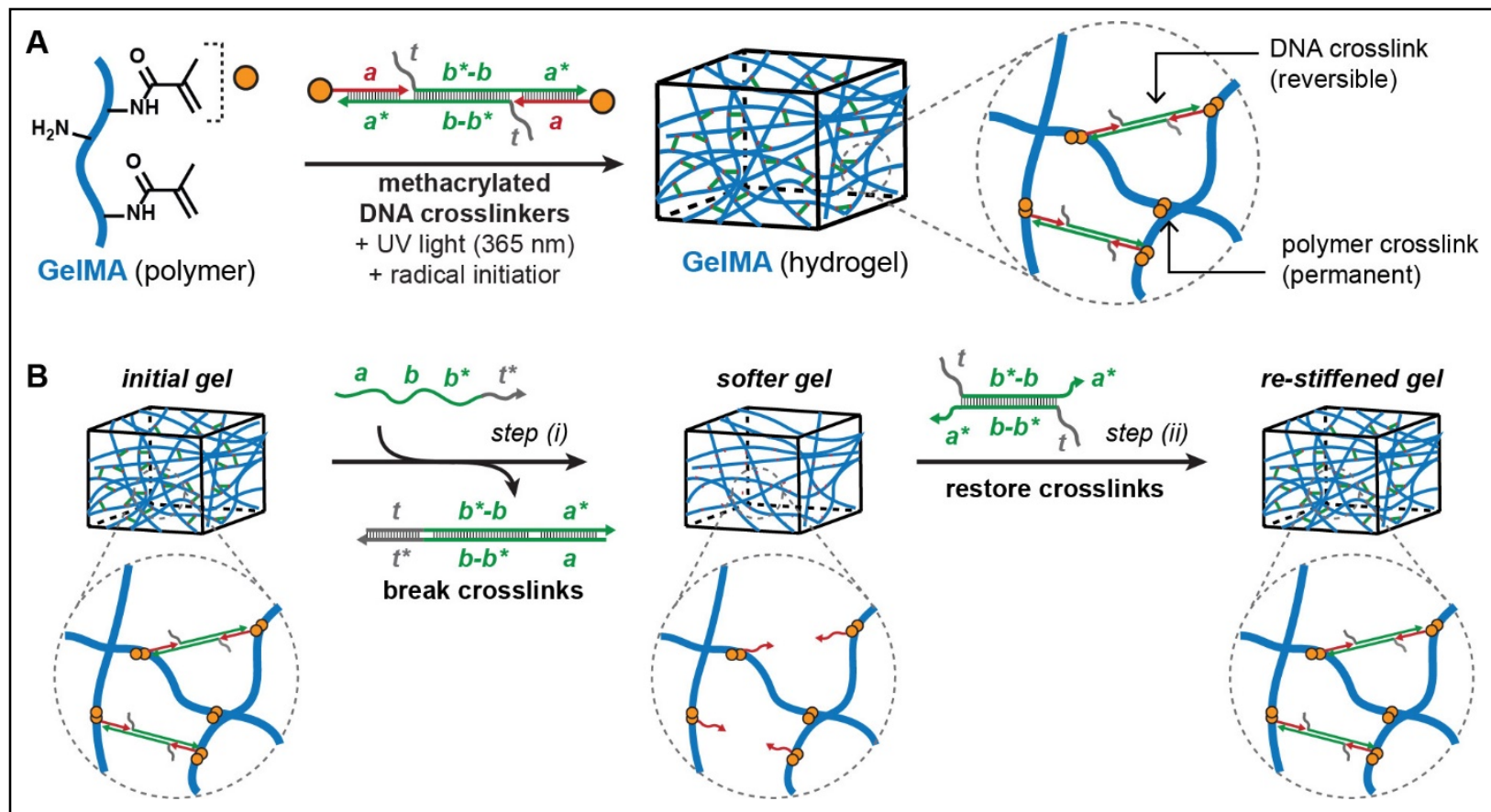

Figure 1. Design of reversible GeIMA-DNA hydrogels. A) Crosslinking gelatin methacrylate (GelMA) polymers with dsDNA crosslinkers functionalized with 5' methacrylamide moieties to give a hydrogel. The gel consists of both reversible DNA-based crosslinks, and permanent crosslinks between GelMA polymers. B) The initial, DNAcrosslinked hydrogel can be softened (step (i)) by the addition of a displacement strand fully complementary to the crosslinks, leaving behind ssDNA handles (red) attached to the GelMA. Adding a fresh batch of dsDNA crosslinker complementary to the tethered handles (step (ii)) restores the crosslinks, and increases the stiffness of the gel.

signals-and tunable reversibility through the use of displacement strands. ${ }^{12}$ For example, DNA has been used as a crosslinker for polymeric hydrogels, ${ }^{13-26}$ or as the bulk of a biomaterial through the use of branched motifs; ${ }^{27-29}$ in both cases DNA-specific properties like strand displacement or enzymatic degradation imbued these materials with dynamic properties. Recently, the Stupp laboratory pioneered the use of DNA to control the presentation of multiple ligands on a biomaterial surface ${ }^{30}$ and demonstrated the hierarchical assembly and mechanical tunability of self-assembled peptide amphiphile nanofibers. ${ }^{31}$ A follow-up work by Freeman and colleagues further extended this principle to Fmoc-based peptide fibers. ${ }^{32}$ However, all of the examples above used either synthetic polymers or engineered self-assembling peptides; to our knowledge the use of DNA to reversibly tune the properties of a hydrogel comprised of naturally-derived ECM proteins has not been demonstrated.

Here, we describe the use of DNA to reversibly modulate the stiffness of a 3D gelatin methacrylate (GelMA) hydrogel over two sequential cycles, as well as to independently control crosslink density and ligand presentation. The modification of gelatin with methacrylate groups on the lysine residues provides the advantage of photopatterning, thereby allowing hydrogels to mimic the spatial architecture observed in various tissues, for example the myocardium and the breast. ${ }^{33}, 34$ Additionally, due to the presence of gelatin molecules, GelMA is biocompatible in nature and naturally contains both RGD and MMP sequences to allow cell adhesion, proliferation, and differentiation, along with cellular enzymatic behavior. ${ }^{35}$ GelMA has been previously utilized for multiple purposes including cardiac tissue engineering, bone tissue engineering, cancer metastasis studies and vascular network engineering. ${ }^{33-38}$ Imbuing GelMA with reversible, temporal control over stiffness and ligand presentation would provide a better understanding of how different cell types modulate their phenotype and gene expression in response to biophysical 

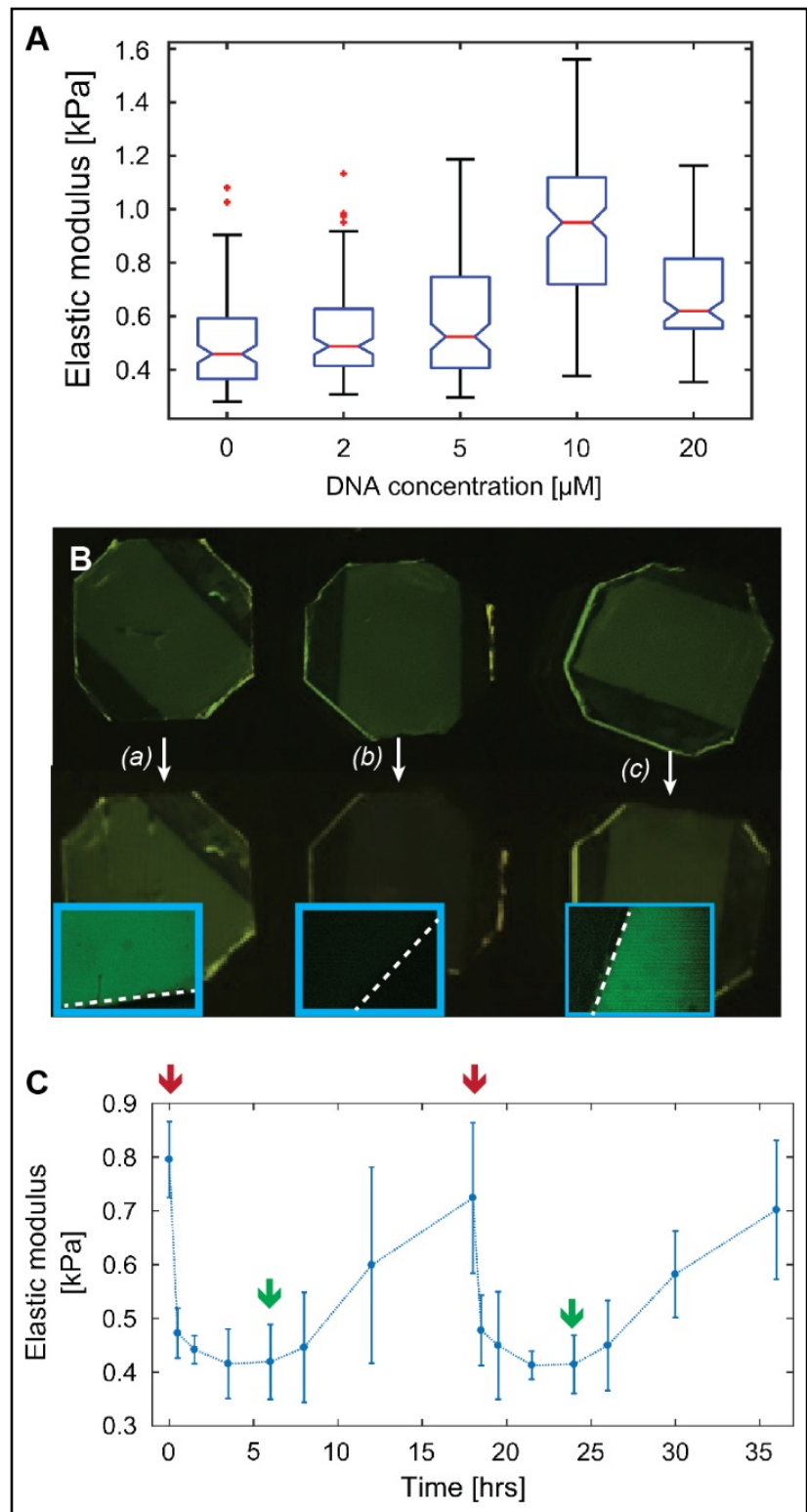

Figure 2. Dynamic control of GelMA crosslinks. A) Elastic modulus (G') of GelMA-DNA hydrogels with different concentrations of crosslinker. B) Probing presence of crosslinker before (top row) and after (bottom row) treatment. Samples: (a), control: no DNA added; $(b)$ addition of displacement strand; (c) addition of mismatched displacement strand. C) Dynamic change in G' over two cycles of displacement and re-stiffening. Red arrows indicate addition of displacement strand and green arrows addition of fresh crosslinker. and biochemical stimuli, which can be of significance in disease modelling such as cancer metastasis studies.

Our design chemically attaches methacrylate moieties to a double stranded (ds) DNA crosslinker. Mixing this crosslinker with GelMA prior to UV-initiated crosslinking gives a self-supporting hydrogel where some of the inter-gelatin crosslinks are replaced by DNA (Figure 1A). By introducing short singlestranded "toehold" sequences (Figure 1A, sequence $\boldsymbol{t}$ (gray)) in the duplex, the crosslink can be broken (Figure 1B, step (i)) by adding a fully complementary displacement strand with sequence $\boldsymbol{a}-\boldsymbol{b}-\boldsymbol{b}^{*}-\boldsymbol{t}^{*}$ (where the asterisk denotes a complementary sequence). After displacement, the methacrylate-linked ssDNA strand (sequence a $(\mathrm{red})$ ) remains attached to the GeIMA, so addition of fresh dsDNA bearing the complementary sequence $\left(\boldsymbol{t}-\boldsymbol{b}^{*}-\boldsymbol{b}-\boldsymbol{a}^{*}\right.$ (green)) restores the crosslinks (Figure 1B, step (ii)). We reasoned that in this fashion, the stiffness of the GelMA hydrogel could be switched between high and low values, over multiple cycles. The triggers for both breaking and re-forming the hydrogel crosslinks are short DNA strands added to the solution bathing the hydrogel, a mild stimulus that is cytocompatible over multiple cycles. ${ }^{30}$ For the design, synthesis, and characterization of all DNA strands used, see Sections S2-S4 and Figures $\mathbf{S 1}$ and $\mathbf{S 2}$ in the Supporting Information. The methacrylamide-modified DNA crosslinker was mixed with high degree methacrylated GelMA (5\% wt/v) followed by exposure to UV light $(360-480 \mathrm{~nm})$ for an interval of 4 seconds as described in Supporting Information to give self-supporting hydrogels (Section S2). We confirmed that the DNA was evenly distributed throughout the hydrogel volume by using a fluorescein (FAM)labeled DNA crosslinker and analyzing the hydrogels using confocal microscopy (Figure S3).

In order to characterize the mechanical properties of the DNA-crosslinked GelMA hydrogels, we used force-indentation experiments with an atomic force microscope (AFM) as previously described. ${ }^{4}, 34,36,39$ We first explored the effect of DNA crosslinker concentration on the elastic modulus ( $G^{\prime}$ ) of the resulting hydrogels (Figure 2A). We found that a crosslinker concentration of 2 or $5 \mu \mathrm{M}$ did not give appreciably different values of $G^{\prime}$ compared with the control $(0.53 \pm 0.18$ and $0.56 \pm 0.23 \mathrm{kPa}$ vs. $0.47 \mathrm{kPa} \pm 0.17$, 


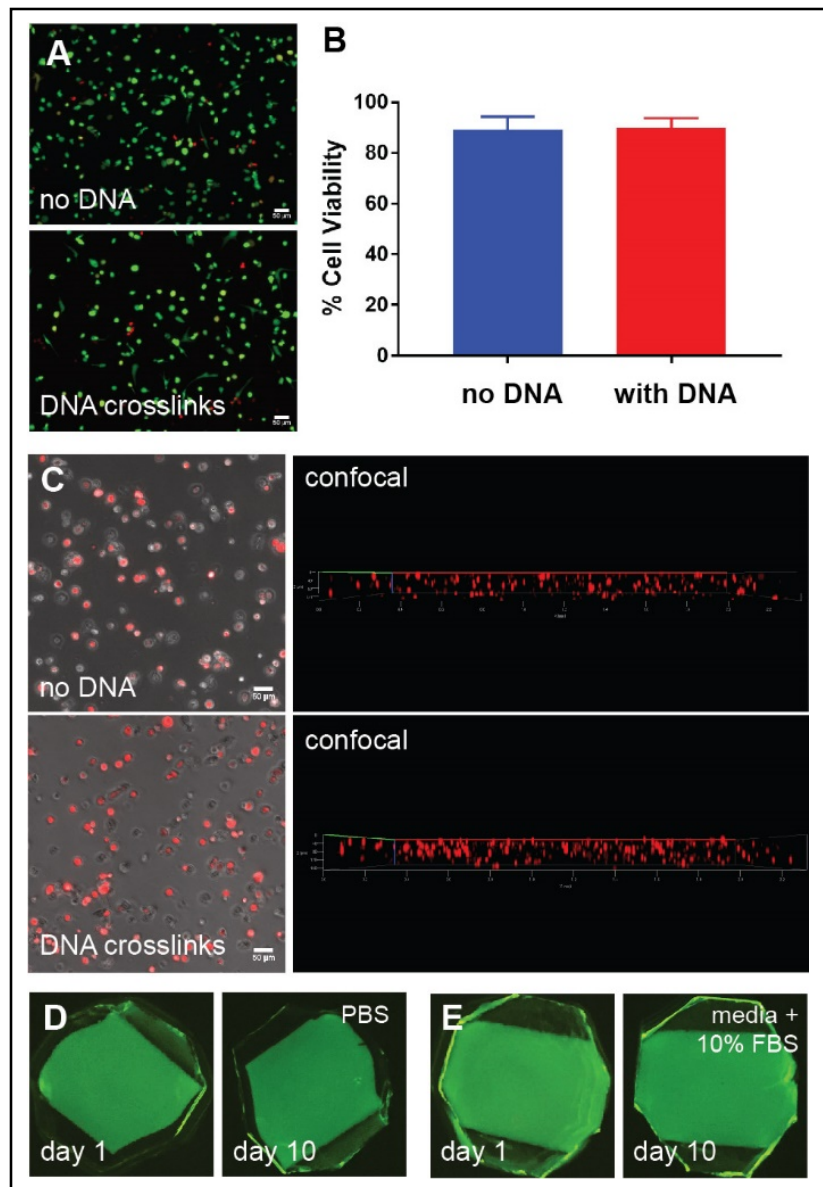

Figure 3. Cell viability and hydrogel stability. A) MDAMB-231 cells in GelMA hydrogels with or without DNA crosslinks. Live cells are green, dead cells are red. B) Quantification of live vs. dead cells after 3 days in culture. C) DsRed-expressing MDA-MB-231 cells in top view and confocal Z-stack to show 3D distribution in hydrogels. D,E) Hydrogels with fluorescent DNA crosslinkers after 1 and 10 days in either PBS (D) or DMEM media $+10 \%$ FBS (E).

Compared with the starting hydrogel stiffness $\left(\mathrm{G}^{\prime}=0.80 \pm .07 \mathrm{kPa}\right)$, addition of displacement strand $(1 \mathrm{mM})$ resulted in a decrease to $\mathrm{G}^{\prime} 0.42 \pm 0.07 \mathrm{kPa}$ in $3.5 \mathrm{~h}$. This corresponds to an almost two-fold reduction in stiffness, with a lower value similar to the control sample without DNA crosslinker added (Figure 2A). After rinsing the displaced crosslinker, we added a fresh batch of crosslinker $(1 \mathrm{mM})$ with ssDNA handles complementary to the handles left behind on the gel. We observed a re-stiffening of the hydrogel over the next $12 \mathrm{~h}$, reaching a peak of $0.72 \pm 0.14 \mathrm{kPa}$, or $\sim 91 \%$ of the initial value. We hypothesize that the increased time for the stiffening process corresponds to the larger size and more rigid nature of the dsDNA crosslinker (compared with the ssDNA displacement strand), which slows its diffusion through the gel. We carried out a second cycle of displacement and re-stiffening, which took roughly the same time as the first cycle (6 and $12 \mathrm{~h}$, respectively), with the final stiffness reaching $0.70 \pm 0.13 \mathrm{kPa}$, or $\sim 97 \%$ compared to the first displacement. To characterize the gel viscoelastic properties we applied dynamic AFM indentation in the frequency range of 1 to $200 \mathrm{~Hz}$. Figure $\mathbf{S 4}$ shows the frequency dependent shear storage (G') and the shear loss moduli (G"), as well as the contact model independent loss 


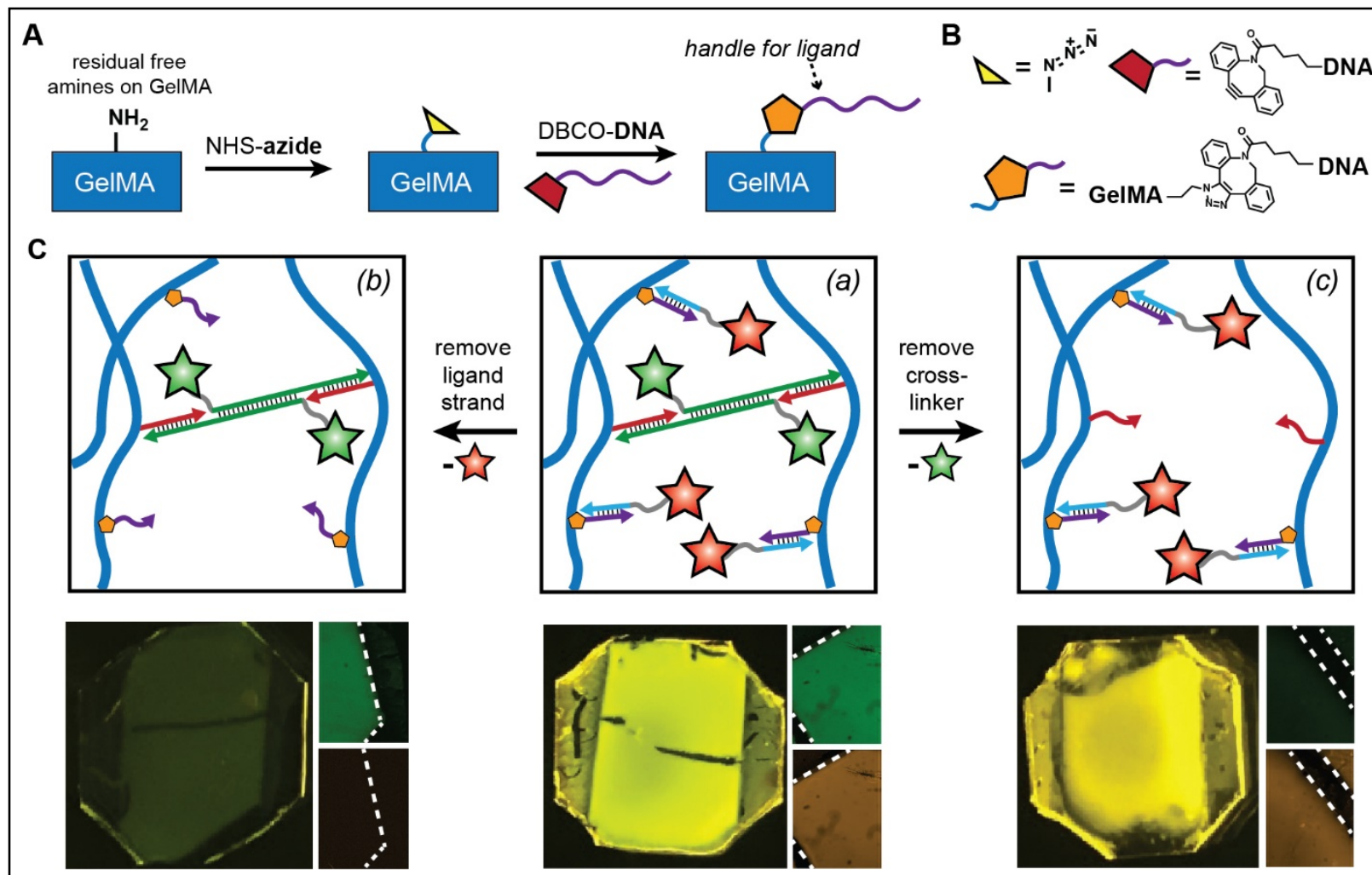

Figure 4. Orthogonal control of ligand presentation and crosslink density. A) Schematic for modification of GeIMA with DNA via copper-free click chemistry with DBCO-DNA. B) Chemical structures for azide (yellow), DBCODNA (red), and triazole product (orange) of the copper-free click reaction between them. C) GelMA modified with a red "ligand" fluorophore (Cy3) and a crosslinker with the green fluorophore FAM ((a), center). Selective displacement of the ligand strand leaves only the green crosslinker $((b)$, left), whereas selective displacement of the crosslinker leaves only the red ligand strand $((c)$, right). Larger images under a UV trans-illuminator, and smaller images were taken under a fluorescence microscope and correspond to the green (top) and red (bottom) channels.

tangent (G'/G'). For all samples and frequencies the loss tangent is smaller than 1 , indicating that the elastic properties of the hydrogels dominate. Taken together, our results demonstrate that DNA strand displacement can reversibly control the stiffness of a GelMA hydrogel over a roughly two-fold range, and that the system does appreciably degrade in performance or speed over two cycles.

To visualize the biocompatibility of the DNA tunable hydrogels, we next incorporated breast cancer MDA-MB-231 cells into the mixture prior to UV irradiation as described in Section S2. After 3 days in culture, we did not observe any difference in survival between cells in GelMA alone compared with cells in gels with $10 \mu \mathrm{M}$ DNA crosslinker ( $89 \pm 5 \%$ vs. $90 \pm 3.9 \%$ ), as probed by a Live/Dead viability assay (Figure 3A,B). The cells were well-distributed throughout the 3D volume of the gels (as probed by confocal microscopy, Figure $3 \mathrm{C}$ using fluorescent tomato red MDA-MB231 cells) and showed no notable morphological differences. One potential concern with oligonucleotide-based biomaterials is that nucleases present in the serum might degrade the DNA over time, which has been observed with a number of DNA structures. ${ }^{40}$ To probe this, we incubated hydrogels with fluorescent DNA crosslinkers with either PBS or DMEM based media containing $10 \%$ fetal bovine serum (FBS). As seen in Figure 3D,E, even after 10 days of incubation no difference in the fluorescence signal was seen, suggesting that the serum does not degrade the crosslinks. This result may be either due to the modification of the termini with methacrylate groups, or due to the tethering of methacrylate groups to the gelatin fibers thereby minimizing nuclease accessibility. 
One of the most challenging aspects in biomaterials research is to control two different types of properties-such as hydrogel stiffness and ligand presentation-in an independent fashion. For example, seminal work by the Anseth lab has demonstrated photo-control of crosslinks and matrix-ligand tethers in polymeric hydrogels using two independent photocleavage reactions. ${ }^{10}$ However, the need for orthogonal photo-chemistries-along with the synthetic challenges in creating these linkers-limits the number of ligands that can be controlled in this fashion. DNA strand displacement, by contrast, can in principle be highly multiplexed by simply changing the sequence of the constituent strands. As a proof-of-principle, we sought to demonstrate that we can elaborate our DNA-crosslinked hydrogels with a second DNA handle of a different sequence. This handle would in turn allow for dynamic control of a model ligand (in this case the fluorophore Cy3) tethered to the complementary strand (Figure 4). Following hydrogel formation as described previously, we appended a second handle to the GelMA by functionalizing free amine residues with azides (via an NHS-PEG4-azide linker) and then coupling them to dibenzocyclooctyne (DBCO)-DNA strand via copper-free click chemistry (Figure 4A,B). ${ }^{41}$ Gels with both DNA strands and fluorophores showed both red and green fluorescence (Figure 4C, sample (a)). Adding a displacement strand for the Cy3 ligand effectively removed the red fluorophore, while leaving the green one intact (Figure 4C, sample (b)). Removing the crosslinker instead yielded a gel with only the red ligand strand (Figure 4C, sample (c)), confirming the orthogonality of the two displacement strands. As an example, the second, "ligand" handle could temporally control the presentation of a growth factor (as previously demonstrated ${ }^{30}$ ), allowing for independent control of ECM stiffness and bioactivity.

In conclusion, we have demonstrated that we can reversibly control the stiffness of a gelatinbased hydrogel using a DNA crosslinker and displacement strands. Our system allows for a roughly two-fold change in elastic modulus, in a reproducible fashion, over two cycles. We note that the changes in stiffness are not particularly dramatic, especially compared with irreversible photocleavage reactions that can degrade the gel completely. ${ }^{9}$ However, the range of $\sim 500-1000$ $\mathrm{Pa}$ accessible is relevant to processes like neural tissue engineering, mesenchymal stem cell differentiation, and breast cancer metastasis. ${ }^{42-44}$ By tuning the design of the crosslinker, it might be possible to obtain a modest increase in the dynamic range of the system. To access dramatically larger values of G' (e.g. 1-2 orders of magnitude) it will most likely be necessary to increase the amount of material inside the hydrogel, as has been recently demonstrated with irreversible, gel-tethered polymerization. ${ }^{45}$ One possible mechanism towards this end is to use DNA-based polymerization mechanisms (such as hybridization-chain reaction ${ }^{13}$ ) in conjunction with protein/peptide -DNA hybrids. ${ }^{46}$ We do, however, highlight that our system is the first to show control over both crosslink density and ligand presentation in the same 3D hydrogel using DNA, and that our method can in principle be extended to orthogonal control of multiple ligand signals. ${ }^{30}$ Although we used gelatin in this report, our approach should be readily extensible to other ECM protein-based hydrogels or synthetic peptide gels as well. Finally, an additional element of control could be introduced into these gels by activating the displacement strand in a spatiotemporal fashion using a photocaged displacement strand ${ }^{47}$ in conjunction with photo-patterning techniques. 


\section{$\underline{\text { References }}$}

1. Walker, C.; Mojares, E.; Hernandez, A. D., Role of Extracellular Matrix in Development and Cancer Progression. International Journal of Molecular Sciences 2018, 19 (10).

2. Lu, P. F.; Weaver, V. M.; Werb, Z., The extracellular matrix: A dynamic niche in cancer progression. Journal of Cell Biology 2012, 196 (4), 395-406.

3. Saini, H.; Eliato, K. R.; Silva, C.; Allam, M.; Mouneimne, G.; Ros, R.; Nikkhah, M., The Role of Desmoplasia and Stromal Fibroblasts on Anti-cancer Drug Resistance in a Microengineered Tumor Model. Cellular and Molecular Bioengineering 2018, 11 (5), 419-433.

4. Saini, H.; Eliato, K. R.; Veldhuizen, J.; Zare, A.; Allam, M.; Silva, C.; Kratz, A.; Truong, D.; Mouneimne, G.; LaBaer, J.; Ros, R.; Nikkhah, M., The role of tumor-stroma interactions on desmoplasia and tumorigenicity within a microengineered 3D platform. Biomaterials 2020, 247.

5. van Helvert, S.; Storm, C.; Friedl, P., Mechanoreciprocity in cell migration. Nature Cell Biology 2018, 20 (1), 8-20.

6. Kai, F.; Drain, A. P.; Weaver, V. M., The Extracellular Matrix Modulates the Metastatic Journey. Developmental Cell 2019, 49 (3), 332-346.

7. Janmey, P. A.; Fletcher, D. A.; Reinhart-King, C. A., STIFFNESS SENSING BY CELLS. Physiological Reviews 2020, 100 (2), 695-724.

8. Badeau, B. A.; DeForest, C. A., Programming Stimuli-Responsive Behavior into Biomaterials. Annual Review of Biomedical Engineering, Vol 21 2019, 21, 241-265.

9. Kloxin, A. M.; Kasko, A. M.; Salinas, C. N.; Anseth, K. S., Photodegradable Hydrogels for Dynamic Tuning of Physical and Chemical Properties. Science 2009, 324 (5923), 59-63.

10. DeForest, C. A.; Anseth, K. S., Cytocompatible click-based hydrogels with dynamically tunable properties through orthogonal photoconjugation and photocleavage reactions. Nature Chemistry 2011, 3 (12), 925-931.

11. Fumasi, F. M.; Stephanopoulos, N.; Holloway, J. L., Reversible control of biomaterial properties for dynamically tuning cell behavior. Journal of Applied Polymer Science 2020, 137 (25).

12. Zhang, D. Y.; Seelig, G., Dynamic DNA nanotechnology using strand-displacement reactions. Nature Chemistry 2011, 3 (2), 103-113.

13. Cangialosi, A.; Yoon, C.; Liu, J.; Huang, Q.; Guo, J. K.; Nguyen, T. D.; Gracias, D. H.; Schulman, R., DNA sequence-directed shape change of photopatterned hydrogels via highdegree swelling. Science 2017, 357 (6356), 1126-1129.

14. Zhao, Z.; Wang, C.; Yan, H.; Liu, Y., Soft Robotics Programmed with Double Crosslinking DNA Hydrogels. Advanced Functional Materials 2019, 29 (45).

15. Li, C.; Zhou, X.; Shao, Y.; Chen, P.; Xing, Y. Z.; Yang, Z. Q.; Li, Z. B.; Liu, D. S., A supramolecular hydrogel with identical cross-linking point density but distinctive rheological properties. Materials Chemistry Frontiers 2017, 1 (4), 654-659.

16. Li, C.; Rowland, M. J.; Shao, Y.; Cao, T. Y.; Chen, C.; Jia, H. Y.; Zhou, X.; Yang, Z. Q.; Scherman, O. A.; Liu, D. S., Responsive Double Network Hydrogels of Interpenetrating DNA and CB 8 Host-Guest Supramolecular Systems. Advanced Materials 2015, 27 (21), 3298-3304.

17. Lin, D. C.; Yurke, B.; Langrana, N. A., Mechanical properties of a reversible, DNAcrosslinked polyacrylamide hydrogel. Journal of Biomechanical Engineering-Transactions of the Asme 2004, 126 (1), 104-110.

18. Li, C.; Faulkner-Jones, A.; Dun, A. R.; Jin, J.; Chen, P.; Xing, Y. Z.; Yang, Z. Q.; Li, Z. B.; Shu, W. M.; Liu, D. S.; Duncan, R. R., Rapid Formation of a Supramolecular PolypeptideDNA Hydrogel for In Situ Three-Dimensional Multilayer Bioprinting. Angewandte ChemieInternational Edition 2015, 54 (13), 3957-3961. 
19. Jiang, F. X.; Yurke, B.; Firestein, B. L.; Langrana, N. A., Neurite outgrowth on a DNA crosslinked hydrogel with tunable stiffnesses. Annals of Biomedical Engineering 2008, 36 (9), 1565-1579.

20. Jiang, F. X.; Yurke, B.; Schloss, R. S.; Firestein, B. L.; Langrana, N. A., The relationship between fibroblast growth and the dynamic stiffnesses of a DNA crosslinked hydrogel. Biomaterials 2010, 31 (6), 1199-1212.

21. Li, C.; Chen, P.; Shao, Y.; Zhou, X.; Wu, Y. Z.; Yang, Z. Q.; Li, Z. B.; Weil, T.; Liu, D. S., A Writable Polypeptide-DNA Hydrogel with Rationally Designed Multi-modification Sites. Small 2015, 11 (9-10), 1138-1143.

22. Zhu, Z.; Wu, C. C.; Liu, H. P.; Zou, Y.; Zhang, X. L.; Kang, H. Z.; Yang, C. J.; Tan, W. $\mathrm{H}$., An Aptamer Cross-Linked Hydrogel as a Colorimetric Platform for Visual Detection. Angewandte Chemie-International Edition 2010, 49 (6), 1052-1056.

23. Wei, B.; Cheng, I.; Luo, K. Q.; Mi, Y. L., Capture and release of protein by a reversible DNA-induced sol-gel transition system. Angewandte Chemie-International Edition 2008, 47 (2), 331-333.

24. Peng, L.; You, M. X.; Yuan, Q.; Wu, C. C.; Han, D.; Chen, Y.; Zhong, Z. H.; Xue, J. G.; Tan, W. H., Macroscopic Volume Change of Dynamic Hydrogels Induced by Reversible DNA Hybridization. Journal of the American Chemical Society 2012, 134 (29), 12302-12307.

25. Guo, W. W.; Lu, C. H.; Qi, X. J.; Orbach, R.; Fadeev, M.; Yang, H. H.; Willner, I., Switchable Bifunctional Stimuli-Triggered Poly-N-Isopropylacrylamide/DNA Hydrogels. Angewandte Chemie-International Edition 2014, 53 (38), 10134-10138.

26. Guo, W. W.; Lu, C. H.; Orbach, R.; Wang, F. A.; Qi, X. J.; Cecconello, A.; Seliktar, D.; Willner, I., pH-Stimulated DNA Hydrogels Exhibiting Shape-Memory Properties. Advanced Materials 2015, 27 (1), 73-78.

27. Um, S. H.; Lee, J. B.; Park, N.; Kwon, S. Y.; Umbach, C. C.; Luo, D., Enzyme-catalysed assembly of DNA hydrogel. Nature Materials 2006, 5 (10), 797-801.

28. Xing, Y. Z.; Cheng, E. J.; Yang, Y.; Chen, P.; Zhang, T.; Sun, Y. W.; Yang, Z. Q.; Liu, D. S., Self-Assembled DNA Hydrogels with Designable Thermal and Enzymatic Responsiveness. Advanced Materials 2011, 23 (9), 1117-1121.

29. Engler, A. J.; Sen, S.; Sweeney, H. L.; Discher, D. E., Matrix elasticity directs stem cell lineage specification. Cell 2006, 126 (4), 677-689.

30. Freeman, R.; Stephanopoulos, N.; Alvarez, Z.; Lewis, J. A.; Sur, S.; Serrano, C. M.; Boekhoven, J.; Lee, S. S.; Stupp, S. I., Instructing cells with programmable peptide DNA hybrids. Nature Communications 2017, 8.

31. Freeman, R.; Han, M.; Alvarez, Z.; Lewis, J. A.; Wester, J. R.; Stephanopoulos, N.; McClendon, M. T.; Lynsky, C.; Godbe, J. M.; Sangji, H.; Luijten, E.; Stupp, S. I., Reversible self-assembly of superstructured networks. Science 2018, 362 (6416), 808-+.

32. Daly, M. L.; Gao, Y.; Freeman, R., Encoding Reversible Hierarchical Structures with Supramolecular Peptide-DNA Materials. Bioconjugate Chemistry 2019, 30 (7), 1864-1869.

33. Saini, H.; Navaei, A.; Van Putten, A.; Nikkhah, M., 3D Cardiac Microtissues Encapsulated with the Co-Culture of Cardiomyocytes and Cardiac Fibroblasts. Advanced Healthcare Materials 2015, 4 (13), 1961-1971.

34. Peela, N.; Sam, F. S.; Christenson, W.; Truong, D.; Watson, A. W.; Mouneimne, G.; Ros, R.; Nikkhah, M., A three dimensional micropatterned tumor model for breast cancer cell migration studies. Biomaterials 2016, 81, 72-83.

35. Sun, M. Y.; Sun, X. T.; Wang, Z. Y.; Guo, S. Y.; Yu, G. J.; Yang, H. Z., Synthesis and Properties of Gelatin Methacryloyl (GelMA) Hydrogels and Their Recent Applications in LoadBearing Tissue. Polymers 2018, 10 (11).

36. Navaei, A.; Saini, H.; Christenson, W.; Sullivan, R. T.; Ros, R.; Nikkhah, M., Gold nanorod-incorporated gelatin-based conductive hydrogels for engineering cardiac tissue constructs. Acta Biomaterialia 2016, 41, 133-146. 
37. Chen, Y. C.; Lin, R. Z.; Qi, H.; Yang, Y. Z.; Bae, H. J.; Melero-Martin, J. M.; Khademhosseini, A., Functional Human Vascular Network Generated in Photocrosslinkable Gelatin Methacrylate Hydrogels. Advanced Functional Materials 2012, 22 (10), 2027-2039.

38. Celikkin, N.; Mastrogiacomo, S.; Jaroszewicz, J.; Walboomers, X. F.; Swieszkowski, W., Gelatin methacrylate scaffold for bone tissue engineering: The influence of polymer concentration. Journal of Biomedical Materials Research Part A 2018, 106 (1), 201-209.

39. Staunton, J. R.; Doss, B. L.; Lindsay, S.; Ros, R., Correlating confocal microscopy and atomic force indentation reveals metastatic cancer cells stiffen during invasion into collagen I matrices. Scientific Reports 2016, 6.

40. Hahn, J.; Wickham, S. F. J.; Shih, W. M.; Perrault, S. D., Addressing the Instability of DNA Nanostructures in Tissue Culture. Acs Nano 2014, 8 (9), 8765-8775.

41. Buchberger, A.; Simmons, C. R.; Fahmi, N. E.; Freeman, R.; Stephanopoulos, N., Hierarchical Assembly of Nucleic Acid/Coiled-Coil Peptide Nanostructures. Journal of the American Chemical Society 2020, 142 (3), 1406-1416.

42. Mohammadi, H.; Sahai, E., Mechanisms and impact of altered tumour mechanics. Nature Cell Biology 2018, 20 (7), 766-774.

43. Ghasemi-Mobarakeh, L.; Prabhakaran, M. P.; Tian, L. L.; Shamirzaei-Jeshvaghani, E.; Dehghani, L.; Ramakrishna, S., Structural properties of scaffolds: Crucial parameters towards stem cells differentiation. World Journal of Stem Cells 2015, 7 (4), 728-744.

44. Pogoda, K.; Chin, L. K.; Georges, P. C.; Byfield, F. J.; Bucki, R.; Kim, R.; Weaver, M.; Wells, R. G.; Marcinkiewicz, C.; Janmey, P. A., Compression stiffening of brain and its effect on mechanosensing by glioma cells. New Journal of Physics 2014, 16.

45. Chan, N. J. A.; Gu, D. Y.; Tan, S.; Fu, Q.; Pattison, T. G.; O'Connor, A. J.; Qiao, G. G., Spider-silk inspired polymeric networks by harnessing the mechanical potential of beta-sheets through network guided assembly. Nature Communications 2020, 11 (1).

46. Stephanopoulos, N., Hybrid Nanostructures from the Self-Assembly of Proteins and DNA. Chem 2020, 6 (2), 364-405.

47. Liu, M. H.; Jiang, S. X.; Loza, O.; Fahmi, N. E.; Sulc, P.; Stephanopoulos, N., Rapid Photoactuation of a DNA Nanostructure using an Internal Photocaged Trigger Strand. Angewandte Chemie-International Edition 2018, 57 (30), 9341-9345. 


\section{Supporting Information}

S1. Materials and Supplies

S2. Methods

S3. DNA sequences Used

S4. Supplementary Figures

S5. References 


\section{S1. Materials and Supplies}

All oligonucleotide strands were purchased from Integrated DNA Technologies (IDT). Strands for methacrylation or azide conjugation were ordered bearing a 5' C6-amine modification. Methacrylic anhydride was purchased from Sigma. NHS-(PEG) $)_{4}$-azide was purchased from Thermo Fisher. NHS-sulfo-DBCO was purchased from Glen Research.

\section{S2. Methods}

DNA Methacrylation: Amine-modified oligonucleotides were dissolved in phosphate buffered saline (PBS) at $\mathrm{pH} 8.0$ to a concentration of $1 \mathrm{mM}$. A 40-fold molar excess of neat methacrylic anhydride was added and the mixture was incubated at RT with agitation for 1 hour. At that point, the reaction was quenched with $15 \mathrm{~mL}$ of water. The solution was then concentrated using a 3 $\mathrm{kDa}$ molecular weight cutoff (MWCO) filter (Amicon).

Synthesis of DNA-DBCO: Amine-modified oligonucleotides were dissolved in phosphate buffered saline (PBS) to a concentration of $1 \mathrm{mM}$. A 5-fold molar excess of DBCO-sulfo-NHS dissolved in DMSO was added to the DNA and agitated at RT overnight. To remove excess $\mathrm{DBCO}$, the reaction mixture was washed three times by buffer exchanging the solution into 50 $\mathrm{mM}$ triethylammonium acetate (TEAA) using a $3 \mathrm{kDa}$ MWCO filter.

HPLC purification of DNA: Modified oligonucleotides were purified using an Agilent 1220 Infinity LC HPLC with a Zorbax Eclipse XDB-C18 column. A linear gradient was generated using $50 \mathrm{mM}$ TEAA/methanol from $10 \%$ to $100 \%$ methanol over 60 minutes. Peak fractions were collected based upon their absorbance at $260 \mathrm{~nm}$, and tested for purity by MALDI-TOF mass spectrometry on an AB SCIEX 4800 MALDI TOF/TOF using a 3-Hydropicolinic acid (HPA) matrix (Sigma). Pure fractions were pooled and buffer exchanged into water using a $3 \mathrm{kDa}$ MWCO filter.

Signal strand surface modification: UV-gelled GeIMA samples were further modified with NHS-(PEG) 4 -azide to functionalize free lysine residues in the hydrogel. GelMA samples were immersed in a freshly prepared $100 \mu \mathrm{M}$ NHS-(PEG) $)_{4}$-azide solution in PBS. The samples were incubated in the reaction solution for 2 hours. Following incubation, the GelMA samples were removed and submersed in fresh PBS to remove any excess free azide. DNA-DBCO conjugates were added to the GelMA samples to a final concentration of $10 \mu \mathrm{M}$ and reacted overnight. The following day the samples were washed with PBS to remove any excess DNA-DBCO. The signal strand was then added and allowed to hybridize at RT for 5 hours in PBS.

Displacement reactions: Displacement reactions were carried out by placing GelMA samples in a solution of the $1 \mathrm{mM}$ displacement strand in PBS. Samples were incubated for 12 hours at RT. Following displacement, samples were imaged on a Zeiss inverted fluorescent microscope (Zeiss Axio Observer Z1) equipped with Apotome 2.0 for Z-stack fluorescence imaging.

Serum Stability experiment: GeIMA samples with FAM-modified crosslinker strands were incubated at RT in DMEM media (VWR Inc.) with 10\% FBS, 1\% L-Glu, 1\% pen/strep and imaged at the appropriate time points on a transilluminator.

Synthesis of Gelatin Methacrylate (GeIMA): Gelatin Methacrylate (GeIMA) was synthesized in a fashion similar to our previous reports. ${ }^{1-3}$ Briefly, to obtain "high-degree methacrylated" GelMA, we utilized $10 \mathrm{~g}$ of Gelatin Type A (Sigma) and dissolved it in $100 \mathrm{~mL}$ of Dulbecco's Phosphate-Buffered Saline $1 \mathrm{X}$ (DPBS $1 \mathrm{X}$ ) at $50{ }^{\circ} \mathrm{C}$ for 1 hour with continuous stirring. Next, methacrylate groups were introduced by addition of methacrylate anhydride (MA) at a 
concentration of $8 \%(\mathrm{v} / \mathrm{v})$ to the prepared gelatin solution. The reaction was allowed to proceed for 3 hours by continuously stirring the mixture followed by addition of $400 \mathrm{~mL}$ of DPBS $1 X$. The solution was dialyzed to remove unreacted MA using 12-14 kDa cut-off dialysis tubing in DI water for 1 week at $40^{\circ} \mathrm{C}$. The dialyzed gel was then aliquoted into $50 \mathrm{~mL}$ centrifuge tubes and stored at $-80{ }^{\circ} \mathrm{C}$ for 24 hours followed by lyophilization for 1 week. The lyophilized GelMA was stored at room temperature for all the experiments.

Fabrication of 3D GeIMA hydrogel samples: To photo-crosslink GeIMA, a 0.5 (w/v)\% solution of photoinitiator ((2-hydroxy-1-(4-(hydroxyethoxy) phenyl)-2-methyl-1-propanone; Sigma) (PI solution) was prepared in 1X DPBS by dissolving at $80^{\circ} \mathrm{C}$. Next, $5 \%$ (w/v) GelMA prepolymer solution was prepared in PI solution and allowed to completely dissolve at $37^{\circ} \mathrm{C}$. For DNA crosslinked samples, preannealed methacrylate crosslinker solution was added to the GeIMA PI solution at the desired final concentration. The GelMA samples were then fabricated by pipetting $15 \mu \mathrm{l}$ of the GelMA pre-polymer solution on top of a spacer with thickness of $100 \mu \mathrm{m}$, followed by inverting onto 3-(trimethoxysilyl) propyl methacrylate (TMSPMA; Sigma) treated glass slides. The whole assembly was then exposed to 4 seconds of UV light ( $800 \mathrm{~mW}, 360-480 \mathrm{~nm})$ followed by removal of glass slides and immediate immersion in 1X DPBS solution to avoid dehydration of hydrogel.

Atomic Force Microscopy (AFM) based indentation experiments: For force-indentation measurements_an Asylum Research MFP-3D-BIO AFM with mesoscopic sphereo-conical (probe radius $\sim 750 \mathrm{~nm}$ ) Team NanoTec LRCH-750 AFM probes were used. The spring constants (nominal $\mathrm{k} \sim 0.2 \mathrm{~N} \cdot \mathrm{m}^{-1}$ ) were determined using thermal energy dissipation method. ${ }^{4,5}$ Samples were measured at $37^{\circ} \mathrm{C}$ in $1 \mathrm{X}$ Hank's Balanced Salt Solution (HBSS) containing calcium and magnesium. The samples were also buffered with $25 \mathrm{mM}$ HEPES to maintain their $\mathrm{pH}$ during the measurements. Quasi-static measurements with cantilever approach and retraction speed 2 $\mu \mathrm{m} \cdot \mathrm{s}^{-1}$ were conducted to collect elastic modulus data. In $90 \mu \mathrm{m} \times 90 \mu \mathrm{m}$ areas two grids of $4 \times 4$ indentations per sample were acquired by applying trigger forces of $20-40 \mathrm{nN}$, which resulted in 10-17 $\mu \mathrm{m}$ of indentation. The first $10 \mu \mathrm{m}$ of the force-indentation curves were fitted to a nonadhesive quasi-static contact model for a canonical indenter with a spherical tip that features continuous curvature at the transition point. ${ }^{6}$ To quantify the temporal mechanical changes over two cycles, samples treated with $10 \mu \mathrm{M}$ crosslinker DNA were used. First, the initial elastic moduli of three samples were measured. Later, the buffer was aspirated, and the gel surface was covered by $60 \mu \mathrm{L}$ of the displacement DNA (at $1 \mathrm{mM}$ concentration) and incubated at $37^{\circ} \mathrm{C}$. In each cycle the elastic moduli were measured within 6 hours of softening. The displacement solution was then replaced by crosslinker solution $(1 \mathrm{mM})$ and the elastic moduli were measured for the 12 hour of stiffening process. The DNA solution was collected before each measurement and the same DNA solution was added to the samples after the measurement was completed and the samples were incubated at $37^{\circ} \mathrm{C}$ again.

Cell encapsulated GelMA samples: To visualize the effect of tunable hydrogels on cancer cells, we utilized tomato red MDA-MB-231 (p16-22) breast cancer cells transduced to express DsRed fluorescence. The breast cancer cells were cultured in DMEM 1X media supplemented with 10\% Fetal Bovine Serum (FBS), 1\% L-Glutamine and 1\% pen/strep. The cells were trypsinized and re-suspended with GelMA pre-polymer solution at a density of $2 \times 10^{6}$ cells per $\mathrm{mL}$. About $15 \mu \mathrm{L}$ of cell-suspended GelMA pre-polymer solution was pipetted on the spacer, on top of which TMSPMA coated glass slides were inverted. The whole assembly was exposed to UV for 12 seconds. The glass slide was then removed and immersed in 1x DPBS followed by submersion in a well of 24 well plate containing $500 \mu \mathrm{L}$ of the media.

Cell viability assay: Cell viability was assessed using a Live/Dead Viability kit (Invitrogen) containing calcein-AM (for staining live cells, green fluorescence) and ethidium homodimer-1 (for 
staining dead cells, red fluorescence). The solution was prepared by adding $0.5 \mu \mathrm{L}$ calcein-AM and $2 \mu \mathrm{L}$ Ethidium homodimer-1 in $1 \mathrm{~mL}$ pre-warmed DPBS. Media was removed from the cellencapsulated GeIMA samples followed by their gentle washing with 1X DPBS without disturbing the gels. Next $150 \mu \mathrm{L}$ of viability solution was added on top of the samples and incubated at $37^{\circ}$ $\mathrm{C}$ for 30 minutes. The samples were then imaged using an Inverted Zeiss Microscope (Zeiss Axio Observer Z1) at 2-3 random locations to acquire fluorescent images at 10x magnification. Since MDA-MB-231 cells were transduced to express DsRed fluorescence, cellular viability was quantified by counting number of live cells (green fluorescence) and total number of cells using cell counter plugin in ImageJ similar to our previous report. ${ }^{3}$ The cell viability was therefore calculated as percentage of viable cells over total number of cells in each image.

\section{S3. DNA sequences}

\begin{tabular}{|c|c|}
\hline Strand & Sequence $\left(\mathbf{5}^{\prime} \rightarrow \mathbf{3}^{\prime}\right)$ \\
\hline Methacrylated DNA strand & 5'-C6-amine-GTCAACGCTCCAGAAC \\
\hline crosslinking duplex & CAAGTACTTCGCAATACGCGTATTGCGAAGTTCTGGAGCGTTG \\
\hline FAM - crosslinking duplex & FAM - CAAGTACTTCGCAATACGCGTATTGCGAAGTTCTGGAGCGTTG \\
\hline crosslinking Displacement strand & CAACGCTCCAGAACTTCGCAATACGCGTATTGCGAAGTACTTG \\
\hline scrambled crosslinking Displacement strand & AATCACCTGACTGATTCACCTCGGGAACGTTTGATAAGCGCCA \\
\hline DBCO DNA strand & 5'-C6-amine-CCATCTGGTATTAC \\
\hline Gelma signal strand & Cy3 - GATATACGTAATACCAGATGG \\
\hline Gelma Signal Disp & CCATCTGGTATTACGTATATC \\
\hline
\end{tabular}




\section{S4. Supplementary Figures}

A
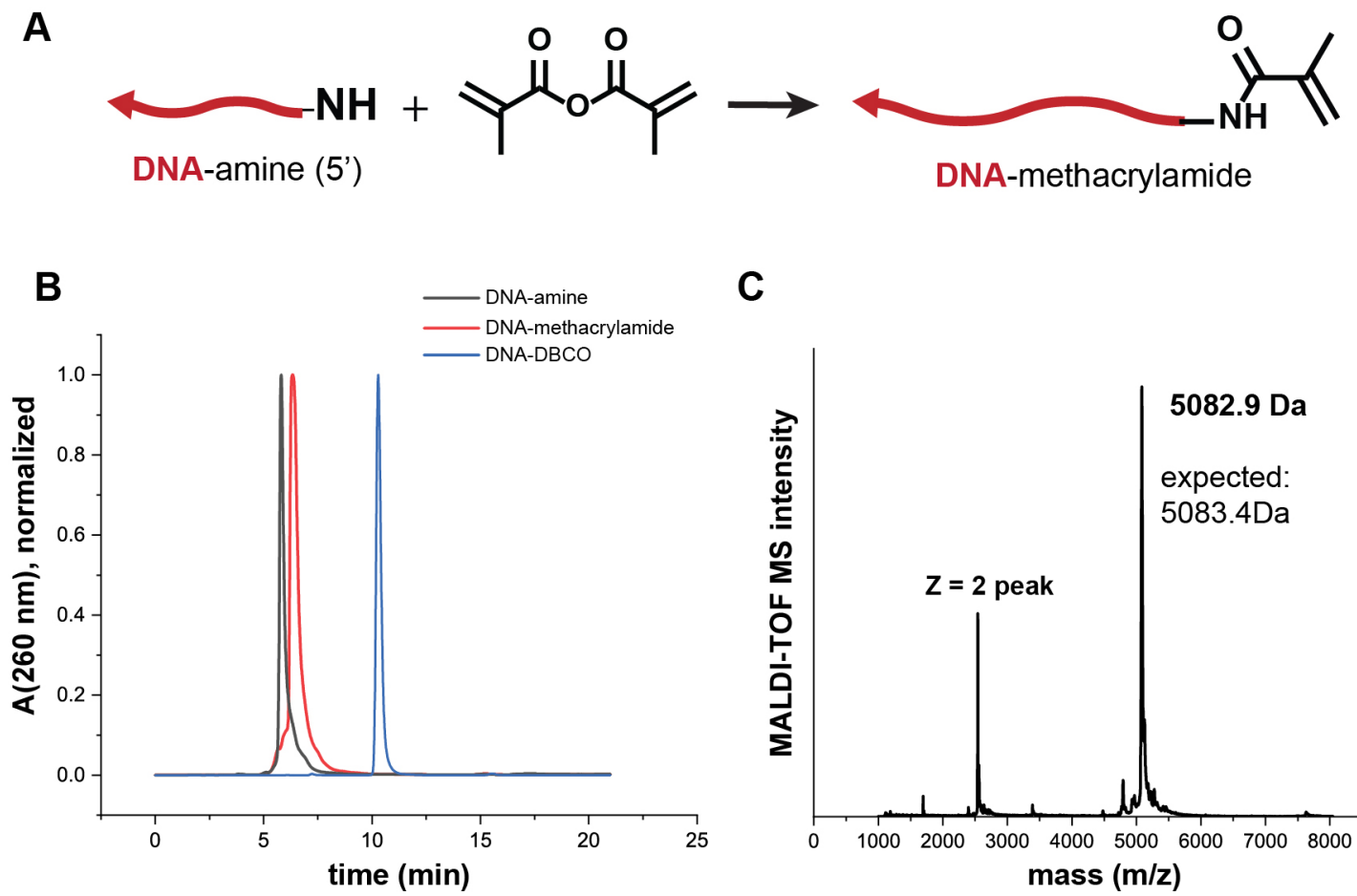

C

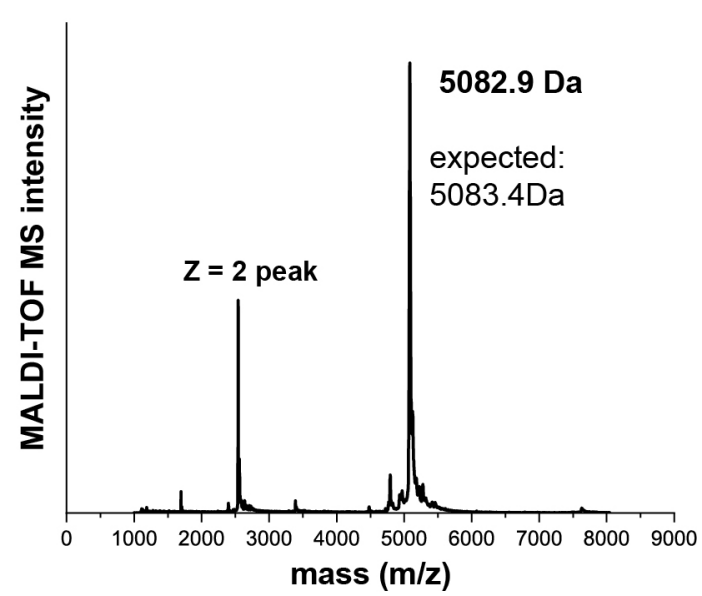

Figure S1: Synthesis and characterization of modified DNA. A) ssDNA handles bearing a 5'amine functionality were modified with methacrylic anhydride to yield DNA-methacrylamide. B) Reverse-phase HPLC traces of the unmodified DNA-amine, as well as the purified DNAmethacrylamide and DNA-DBCO. C) MALDI-TOF mass spectrum of the DNA-methacrylamide. 
A

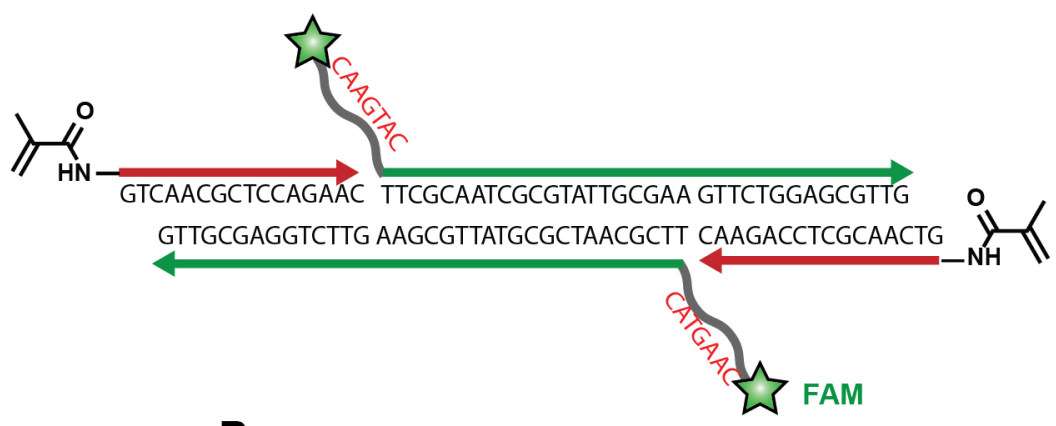

B

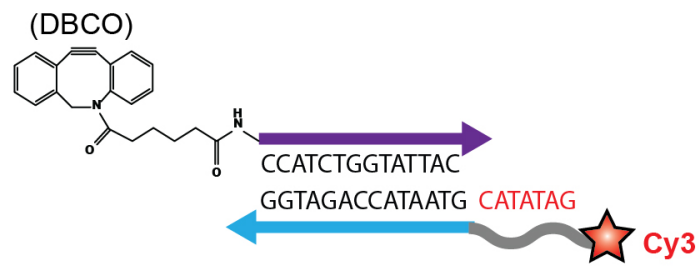

C

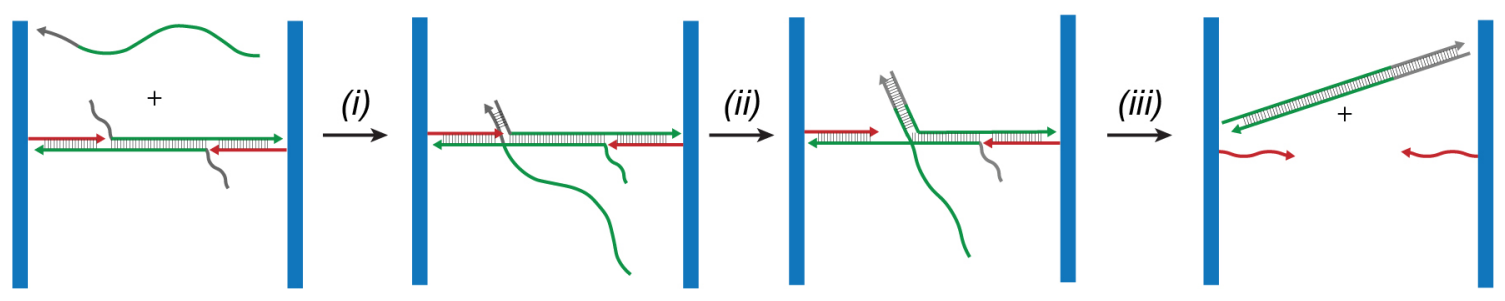

Figure S2: DNA design and strand displacement. A) Design of crosslinker strand, showing sequences, toeholds (red letters) and attachment of green FAM fluorophore. B) Design of "ligand" strand, showing sequences, toehold (red letters) and attachment of red Cy3 fluorophore as a model ligand. C) Detail of strand displacement process, showing toehold binding (i), branch migration (ii), and crosslinker duplex release (iii). For DNA strands, arrows point in the $5^{\prime} \rightarrow 3$ ' direction.
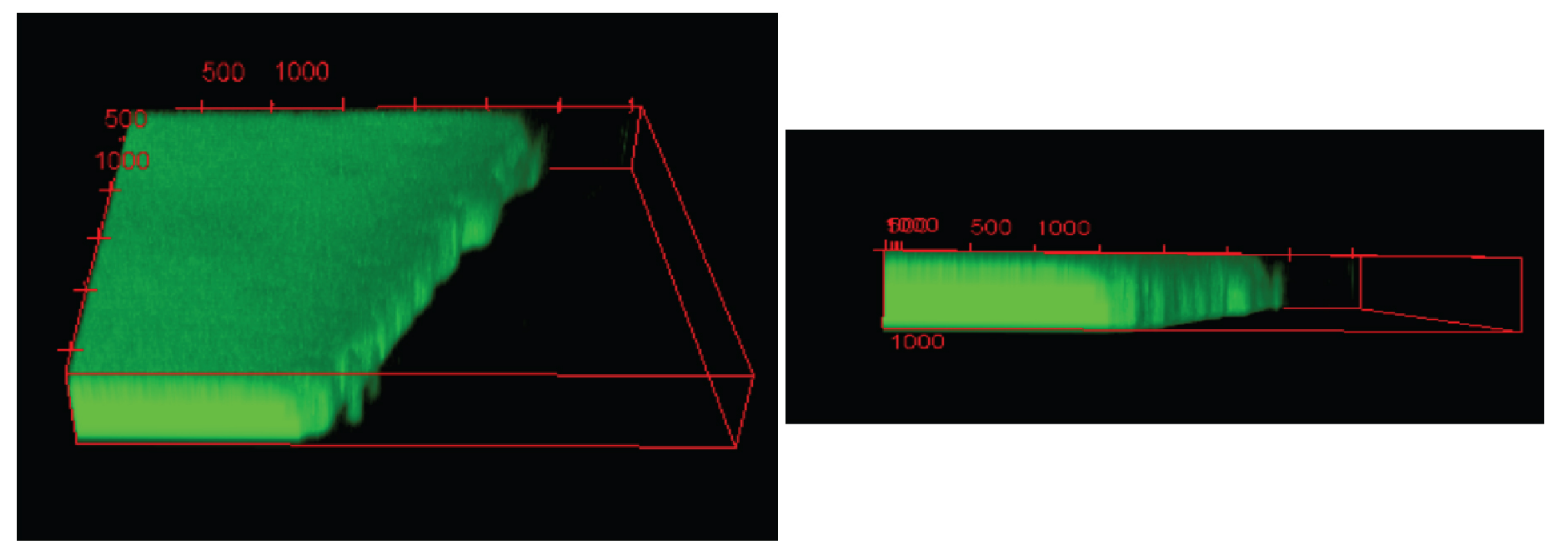

Figure S3: 3D reconstruction of gel volume. Two views of the DNA-crosslinked GeIMA hydrogel, showing that the FAM-labeled crosslinker is evenly distributed throughout the entire volume. 

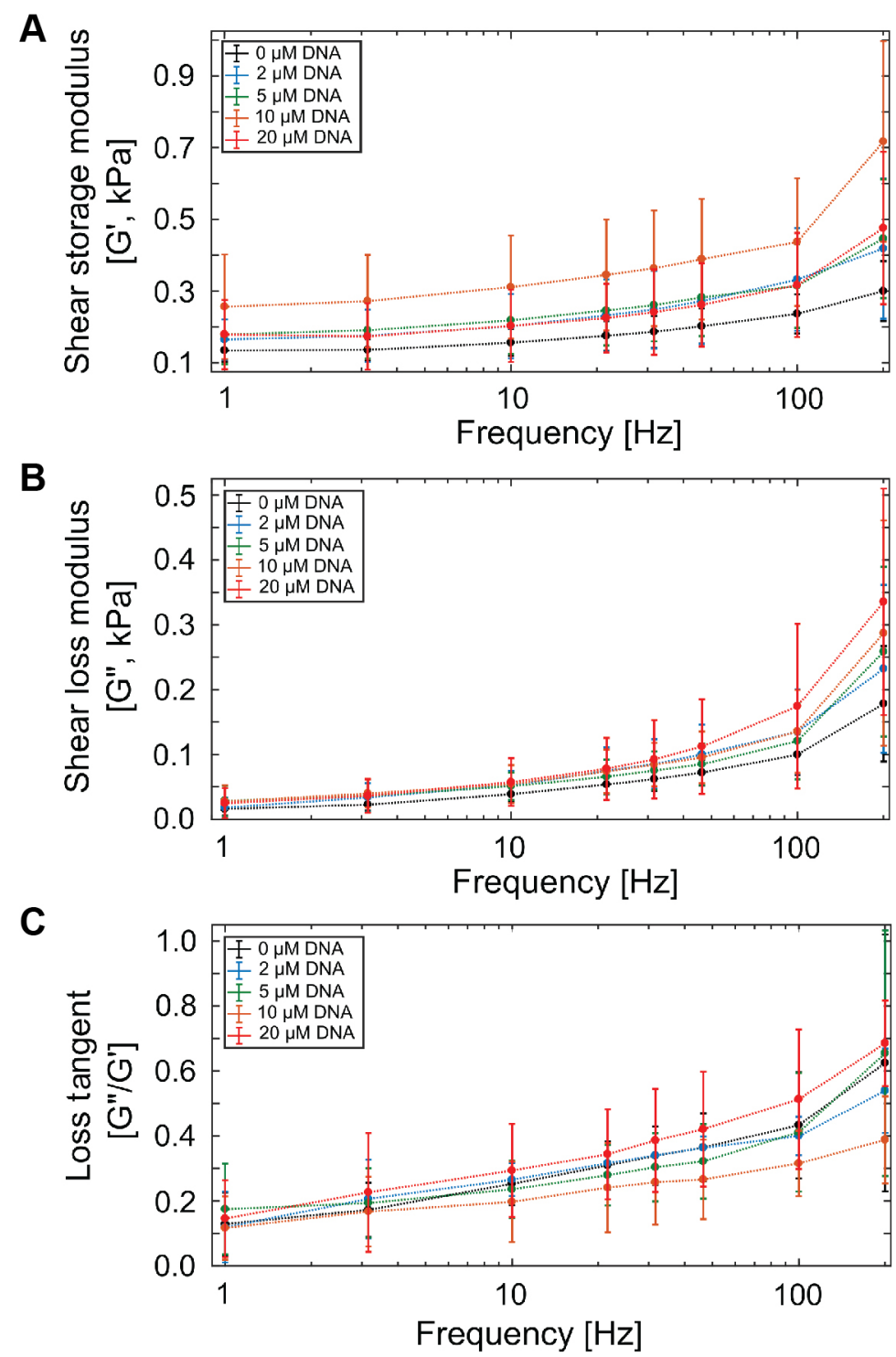

Figure S4: Dynamic properties of hydrogels. A) Frequency dependent shear storage (G'). B) Shear loss moduli (G”). C) Loss tangent (G'/G”). 


\section{S5. References}

1. Saini, H.; Navaei, A.; Van Putten, A.; Nikkhah, M., 3D cardiac microtissues encapsulated with the co-culture of cardiomyocytes and cardiac fibroblasts. Adv Healthc Mater 2015, 4 (13), 1961-71.

2. Navaei, A.; Saini, H.; Christenson, W.; Sullivan, R. T.; Ros, R.; Nikkhah, M., Gold nanorodincorporated gelatin-based conductive hydrogels for engineering cardiac tissue constructs. Acta Biomater 2016, 41, 133-46.

3. Peela, N.; Sam, F. S.; Christenson, W.; Truong, D.; Watson, A. W.; Mouneimne, G.; Ros, R.; Nikkhah, M., A three dimensional micropatterned tumor model for breast cancer cell migration studies. Biomaterials 2016, 81, 72-83.

4. Hutter, J. L.; Bechhoefer, J., CALIBRATION OF ATOMIC-FORCE MICROSCOPE TIPS (VOL 64, PG 1868, 1993). Review of Scientific Instruments 1993, 64 (11), 3342-3342.

5. Butt, H. J.; Jaschke, M., CALCULATION OF THERMAL NOISE IN ATOMIC-FORCE MICROSCOPY. Nanotechnology 1995, 6 (1), 1-7.

6. Staunton, J. R.; Doss, B. L.; Lindsay, S.; Ros, R., Correlating confocal microscopy and atomic force indentation reveals metastatic cancer cells stiffen during invasion into collagen I matrices. Scientific Reports 2016, 6. 